\title{
Anestesia intratecal con morfina por punción única en el hospital san josé: complicaciones y analgesia postoperatoria
}

\author{
Manuel Lorca1, Diego González², Andrea Vega²
}

Introducción: La anestesia intratecal con morfina por punción única se asocia a un riesgo dosis dependiente de depresión respiratoria, con una incidencia entre 0,03 - 7\%. Esta complicación puede asociarse a morbilidad significativa. Recientemente la American Society of Anesthesiology entregó recomendaciones para su uso seguro con un enfoque centrado en esta complicación. En el Hospital San José el uso de morfina intratecal es una modalidad de analgesia postoperatoria utilizada frecuentemente, existiendo un protocolo para su manejo postoperatorio.

Objetivos: El objetivo primario de este trabajo es describir la incidencia de aparición depresión respiratoria en pacientes sometidos a anestesia intratecal con morfina por inyección única. Como objetivo secundario está pesquisar otras complicaciones como náuseas y vómitos post operatorios y prurito, y evaluar lo adecuado de la analgesia según Escala Visual Análoga (EVA).

Material y Métodos: Se obtuvieron las hojas de registro de seguimiento de dolor post operatorio de la unidad de dolor de pabellón central Hospital San José entre las fechas 6/11/2014 y el 7/08/2018. Se consideró depresión respiratoria cualquier episodio descrito como tal por el profesional realizando el seguimiento. Se consideró un nivel de analgesia adecuado cuando la EVA era < 4. Se vaciaron los datos a una hoja de cálculo (Microsoft Excel 2016) y se procedió a su tabulación. Se eliminaron pacientes cuyos datos estaban incompletos.

Resultados: Se documentaron 110 anestesias intratecales con morfina. El promedio de edad fue 59,48 $\pm 16,30$. La distribución por sexo fue 33 hombres y 69 mujeres. Se realizaron 101 anestesias con morfina intratecal y sólo 1 con morfina + fentanil intratecal. Ningún paciente presentó depresión respiratoria.
Cuatro pacientes refirieron prurito y 18 pacientes refirieron náuseas y/o vómitos post operatorios (Figura 1). El dolor según EVA al día siguiente de la cirugía fue de 1,6 $2 \pm 2,0$ en reposo y 3,1 $\pm 2,4$ al movimiento. 17 y 38 pacientes experimentaron dolor con EVA $\geq 4$ en reposo y al movimiento respectivamente (Figura 2 ).

Conclusiones: La depresión respiratoria es una complicación muy temida de la anestesia intratecal con morfina. Nosotros no tuvimos ningún caso de depresión respiratoria en 36 meses. La incidencia más alta fue de náuseas y vómitos post operatorios seguido de prurito. El nivel de analgesia postoperatoria en reposo fue adecuado, no siendo satisfactorio la analgesia del dolor gatillado por movimiento. Tomando las precauciones necesarias incluyendo la monitorización post operatoria el uso de anestesia intratecal con morfina es una buena alternativa de analgesia postoperatoria.

\section{Referencias}

1. Hindle A. Intrathecal opioids in the management of acute postoperative pain. Contin Educ Anaesth Crit Care Pain. 2008;8(3):81-5.

2. Practice Guidelines for the Prevention, Detection, and Management of Respiratory Depression Associated with Neuraxial Opioid Administration: An Updated Report by the American Society of Anesthesiologists Task Force on Neuraxial Opioids and the American Society of Regional Anesthesia and Pain Medicine. Anesthesiology. 2016 Mar;124(3):535-52.

3. Sultan P, Gutiérrez MC, Carvalho B. Neuraxial morphine and respiratory depression: finding the right balance. Drugs. 2011 Oct;71(14):1807-19.

Hospital San José.

Universidad Santiago de Chile. 